\title{
Comment on: Surgery and HIPEC in Recurrent Epithelial Ovarian Cancer: A Prospective Randomized Phase III Study
}

\author{
Thales Paulo Batista, MD, MS ${ }^{1,2}$ \\ ${ }^{1}$ Department of Surgery/Oncology, Faculdade Pernambucana de Saúde, Instituto de Medicina Integral Professor Fernando \\ Figueira - FPS/IMIP, Recife, PE, Brazil; ${ }^{2}$ Department of Gynecology, Hospital de Câncer de Pernambuco - HCP, Recife, \\ PE, Brazil
}

\section{TO THE EDITORS,}

Hyperthermic intraperitoneal chemotherapy (HIPEC) is certainly a promising treatment option for patients with advanced ovarian cancer, since this disease often remains limited to the peritoneal cavity and this is also the preferred site of recurrence. However, there is insufficient evidence in scientific literature to advocate this approach as a formal therapy for ovarian cancer, and HIPEC still needs to be evaluated by means of randomized trials, which may surely provide additional information on this matter. In this setting, we read with great interest the recently published article by Spiliotis et al. in Annals of Surgical Oncology ${ }^{1}$ describing the first phase III trial exploring use of HIPEC for ovarian cancer.

Despite the scientific merit of this pioneering study, we noticed some drawbacks in the presentation that we would like to point out as follows: Firstly, I believe that the statistical analysis was not clearly described and applied. The authors mainly focused their presentation on "mean overall survival," whereas the survival estimation was also presented as Kaplan-Meier plots and (probably) compared by the log-rank test. Another main point is that the median survival was not reached during the follow-up period according to the survival plots presented in the article, an important fact that was not mentioned by the authors. Accordingly, to present survival rates as "means" is probably not appropriate to a nonparametric approach (i.e., Kaplan-Meier plus log-rank test) as presented. Instead of

(C) Society of Surgical Oncology 2017

First Received: 13 October 2017;

Published Online: 1 November 2017

T. P. Batista, MD, MS

e-mail: t.paulo@outlook.com that, the authors should base their survival analysis on (cumulative proportional) progression-free survival, especially because this is an excellent predictor of later overall survival. The only reference to this type of presentation is in the abstract, and the reported "3-year survival" rates of $75 \%$ versus $18 \%$ appear to be not in accordance with the survival plots presented in the results (i.e., Fig. 1, no HIPEC group). Finally, the subgroup analyses were mitigated by the variable and shorter follow-up, while I am not sure that the sample size calculation was powered to permit appropriate conclusions.

In conclusion, I would like to congratulate the authors for their efforts in developing this phase III trial, and for the discussion of their interesting results in regard to survival rates between patients with platinum-resistant versus platinum-sensitive disease. However, in view of the criticism presented above, we feel that no definitive conclusion is possible based on their data, and further studies are necessary before HIPEC may be considered a formal treatment option for recurrent ovarian cancer. We are currently eagerly waiting results of other ongoing phase III trials.

DISCLOSURE None.

\section{REFERENCE}

1. Spiliotis J, Halkia E, Lianos E, Kalantzi N, Grivas A, Efstathiou E, Giassas S. Cytoreductive surgery and HIPEC in recurrent epithelial ovarian cancer: a prospective randomized phase III study. Ann. Surg. Oncol. 2014;22:1570-5. 